\title{
Understanding the spatial mass transfer behaviour of a pulsating jet HMV system - vortex generation and characterisation
}

\author{
Peter R. Birkin ${ }^{1 a}$, Jekaterina Kuleshova ${ }^{a}$ and Joanne M. Elliott ${ }^{b}$ \\ ${ }^{a}$ Chemistry, University of Southampton, Southampton, United Kingdom Fax: +44 \\ (0)2380593781; Tel: +44(0)2380594172; E-mail: prb2@soton.ac.uk
}

${ }^{b}$ Department of Chemistry, University of Reading, Reading, United Kingdom Fax:

+44(0)118 378 6331; Tel: +44(0)118 378 6342; E-mail: j.m.elliott@reading.ac.uk

\begin{abstract}
The flow patterns generated by a pulsating jet used to study hydrodynamic modulated voltammetry (HMV) are investigated. It is shown that the pronounced edge effect reported previously is the result of the generation of a vortex ring from the pulsating jet. This vortex behaviour of the pulsating jet system is imaged using a number of visualisation techniques. These include a dye system and an electrochemically generated bubble stream. In each case a toroidal vortex ring was observed. Image analysis revealed that the velocity of this motion was of the order of $250 \mathrm{~mm} \mathrm{~s}^{-1}$ with a corresponding Reynolds number of the order of 1200 . This motion, in conjunction with the electrode structure, is used to explain the strong 'ring and halo' features detected by electrochemical mapping of the system reported previously.
\end{abstract}

\footnotetext{
${ }^{1}$ Corresponding author
} 


\section{Introduction}

The investigation of the characteristics of a pulsating jet[1-3] for hydrodynamic modulated voltammetry (HMV)[4-17] applications has been reported in a number of previous articles. In brief, the apparatus (an inverted funnel and a narrow $(\sim 2 \mathrm{~mm}$ in diameter) jet orifice, produces a reproducible hydrodynamic pulse of liquid which can, in combination with electrochemical apparatus, be useful. For example, this approach has been used to investigate HMV behaviour of a variety of redox systems[2, 3, 18]. In addition, the detection limit of the system[3] and its use to investigate nanostructured[19-21] electrodes has been demonstrated[1]. However, in the course of previous investigations, mapping of the electrochemical response of the system identified some unusual characteristics[2]. First, the current pulses produced by this apparatus exhibited a strong 'ring' like relationship with the edge of the jet mouth[2]. Second, the size of the electrode surround (or support) also affected the mapping data, with a 'halo' effect detected. Third, the HMV response of the jet could be detected at a considerable distance $(\sim 1 \mathrm{~cm})$ from the mouth of the jet. While these observations do not affect the operation of the apparatus as a HMV system, they do require further explanation. This is the subject of this manuscript. In order to investigate these observations further, a set of experiments were performed to image the flow patterns produced by the pulsating jet. There are many examples in the literature [22-25] (see ref [25] for general examples) where flow visualisation has been achieved through the use of a variety of different approaches, the experiments described here utilised dye species and gas bubble generation in efforts to visualise the flow of material from the jet into the bulk solution and then onto an electrode support[25, 26]. These two techniques were chosen as they enabled detailed imaging of the first pulse (in the case of the dye 
system) or an investigation of the repeated fluid flow as a result of the pulsating jet (in the case of the bubble tracer method). In addition it should be noted that the flow generated in this system is periodic in nature. A brief discussion of these techniques and the results of experiments performed on the pulsating jet system will now be presented. 


\section{Materials and Methods}

The experimental arrangement used in this work and specifically its operation to gather useful hydrodynamic modulated voltammetric data has been reported previously. The modulated jet was generated by forced oscillation of a $\sim 3.5 \mathrm{~cm}$ radius membrane attached to the base of an inverted funnel. The neck of the funnel was then pulled into a $2 \mathrm{~mm}$ diameter jet orifice. Oscillation of the membrane was driven by a mechanical shaker (a Model V4, Signal Force, Data Physics Ltd) attached to the centre of a piston/membrane assembly (note although the piston assembly provided significantly improved HMV performance when compared to the membrane system, both will be used in the results discussed here). Figure 1 shows a schematic of a section of the pulsating jet apparatus imaged in the experiments reported here with particular reference to the region of direct interest where the imaging and electrochemical scanning experiments where concentrated[2]. Note variants of the complete apparatus can be found described previously in more detail [1-3]. In order to measure the displacement of the base of the apparatus, the mechanical coupling between the shaker and the membrane or membrane/piston assembly incorporated a single axis accelerometer[18] (Model 3100B, Dytran Instruments, Inc., sensitivity $99.3 \mathrm{mV} \mathrm{g}^{-1}$ ). This enables access to the magnitude of motion of the membrane/piston which in turn can be used to estimate the fluid flow at the jet orifice. In order to achieve this estimation the signal from the accelerometer was conditioned with an amplifier (Model 4105C, Dytran Instruments, Inc.) and recorded on an oscilloscope (Techtronixs, TDS 2014, 1GS/s, $100 \mathrm{MHz}$ ). The shaker was driven using a Signal Force 30W Power amplifier, Model PA 30E, Data Physics Ltd) which is in turn supplied by a function generator (TGA12101 $100 \mathrm{MHz}$ Arbitrary Waveform generator, TTi Ltd). In all cases the frequency of oscillation of the 
membrane/piston assembly, and hence jet, was set to $16 \mathrm{~Hz}$. The zero-to-peak displacement amplitude of the centre of the piston or membrane is stated in the appropriate figure legend. Positioning of the electrode with respect to the jet orifice was controlled using an XYZ stage (Zaber, $60 \mathrm{~mm}$ travel, resolution $0.1 \mu \mathrm{m}$, TLA 60A actuators connected to TSB 60-M stages). Figure 1 shows a schematic of the apparatus used in the imaging experiments. Two approaches to the visualisation of the flow from the jet were employed. First, the entire volume within the inverted funnel was filled with water coloured with a dye. The outer volume was then carefully filled with pure water. Imaging of the flow was then performed as the shaker was activated. Typically the first pulse only was recorded using this method. Second, a Pt microwire (50 $\mu \mathrm{m}$ in diameter, Advent research materials) was incorporated in the neck of the inverted funnel. In order to do this, the glass funnel was first cut $\sim 1 \mathrm{~cm}$ from the jet exit and the $50 \mu \mathrm{m}$ diameter Pt wire glued using fast set epoxy resin, laterally across the neck of the main body of the funnel. Finally the jet exit was reattached using fast set epoxy to complete the flow apparatus. Care was taken to ensure that the glue did not protrude into the flow path within the neck of the inverted cone. In addition the choice of the $50 \mu \mathrm{m}$ diameter wire also ensures that as little perturbation as possible on the flow in this region was generated. Note that this is supported by the close correlation between dye and bubble tracer experiments (see later images). Figure 1 also shows the region imaged using a high-speed camera in order to understand the flow generated in more detail. Electrochemical bubble generation was then achieved by applying a suitable current controlled with a voltage source (Maplin) between the Pt microwire and a Pt gauze electrode placed in the upper chamber of the pulsating jet system. A Photron APX RS high-speed camera with a Navitar $12 x$ lens was used to record the high-speed 
images. In these experiments the apparatus was backlit and side lit with a LED light source and $20 \mathrm{~W}$ halogen lamp respectively. Solutions were prepared using a Purite Select (Ondeo) water purification system (resistivity typically $>15 \mathrm{M} \Omega \mathrm{cm}$ and a TOC $<10 \mathrm{ppb}$ according to the manufacturer). $\mathrm{Sr}\left(\mathrm{NO}_{3}\right)_{2}$ (Aldrich, $99+\%$ ) and red food dye (Super Cook) were used as received. The solutions were at room temperature (20$25^{\circ} \mathrm{C}$ ) and the experiments were performed under aerobic conditions. 


\section{Results and discussion}

Figure 2 shows a set of images taken, with respect to time, of the neck of the pulsating jet (note the solid black line towards the top of each image in (a) represents the air/liquid interface). In this case the funnel section of the apparatus was filled with water coloured with a red dye while the upper chamber was filled with purified water (see figure 1). In this case some loss of the dyed volume is seen at the neck of the jet itself (see figure $2(a), A 1)$. The mini-shaker was initiated and the region around the neck of the jet imaged. Under these conditions liquid can be seen to be ejected from the jet exit as expected and is seen to emerge from frame A2 onwards. However, the shape of the ejected plume is of interest. The edges of the plume can be seen to wrap around to form a toroidal vortex which then travels through the liquid[27, 28]. The diameter of the toroid $\left(d_{v}\right.$, see figure $\left.2(b)\right)$ is approximately 2.7 $\mathrm{mm}$ but increases from inception at the jet itself until it strikes the air/water interface after frame D5. The velocity of the vortex as it travels through the liquid can also be estimated. For example, considering frames A3-D3 and A5-D5, it is possible to calculate the velocity in these regions as $23.8 \mathrm{~cm} \mathrm{~s}^{-1}$ and $20.6 \mathrm{~cm} \mathrm{~s}^{-1}$ respectively. At the same time as the vortex loses velocity, as it travels through the liquid, measurements of the outer diameter indicate that it has grown from $3.81 \mathrm{~mm}$ to 4.28 $\mathrm{mm}$ for frames A3 to D5 respectively. The motion, loss in velocity and the increase in size of the vortex are all well-known properties of this phenomenon. Figure 2(b) shows one image (specifically C5) expanded to highlight some of the structure seen in the images. The vortex itself can be clearly seen at the top of the dye coloured column. Clear concentric rings which correspond to the fluid motion in the vortex itself can be seen. The value of $d_{v}$ for this vortex is $0.27 \mathrm{~cm}$. In addition a trailing wake of liquid leading back to the jet exit is clearly visible. Figure 2(b) also shows 
that fresh liquid is being drawn back into the jet mouth. This is highlighted on the image as two arrows indicating liquid ingress into the mouth of the jet itself. This occurs through the pulsating nature of the apparatus used. In this particular case a frequency of $16 \mathrm{~Hz}$ is used for the jet frequency. Hence one would expect that for fluid ingress into the mouth of the jet, a time period of $\sim 31.25 \mathrm{~ms}$ will have expired after initiation of the shaker. Close inspection of image D4 C5 shows the first clear evidence of fluid ingress back into the funnel section of the apparatus. This is $34 \mathrm{~ms}$ after the apparatus was initiated in reasonable agreement with the predicted time period considering the uncertainties involved in initiation of the apparatus (response time delay etc.). The measurements made using these high-speed images allow for the Reynolds number $(\mathrm{Re})$ for the vortex to be calculated. Using the approach

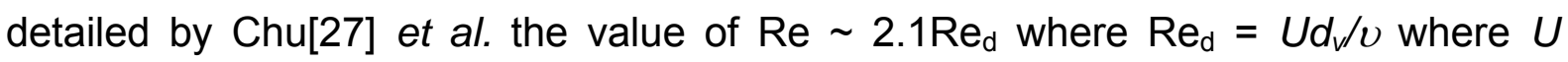
represents the velocity of the vortex and $v$ the kinematic viscosity of the media[27]. Analysis of the images shown in figure 2 shows that under these conditions the value of $U$ was $20.6 \mathrm{~cm} \mathrm{~s}^{-1}$ (see images A5-D5) while the diameter of the vortex, $d_{v}$, was $\sim 0.27 \mathrm{~cm}$. Using these parameters and assuming a kinematic viscosity of water[29] of the order of $0.01 \mathrm{~cm}^{2} \mathrm{~s}^{-1}$, the value of $\operatorname{Re}$ is $\sim 1168$ for the vortex shown in figure 2(b).

While these images are extremely useful in assessing the shape of the fluid flow leaving the jet during its operation, this fluid dye technique has some limitations. First, in this system only the first ejection of liquid and vortex formation can be seen. Subsequent jet action was less clear as fresh fluid was drawn into the mouth of the jet and then ejected. Second, it is difficult to image the flow patterns precisely within the vortex itself using the dye technique. As an alternative method a bubble tracer system was deployed in the apparatus. Here a $50 \mu \mathrm{m}$ diameter Pt microwire was 
incorporated in the neck of the apparatus (see figure 1 for the position of the microwire with respect to the exit of the jet). This required the jet section of the inverted funnel to be removed and the microwire glued horizontally across the circular section of the neck $(\sim 1 \mathrm{~cm}$ from the jet itself and not shown in the highspeed images to follow). The tip (jet) was then reattached to the funnel to complete the system. This apparatus allowed for bubbles to be generated electrochemically from the wire. These gas bubbles could then be imaged when the pulsating jet was in motion. Note in order to do this the system was lit from both the back and the side. This was found to improve the clarity of the images obtained. Figure $3(a-d)$ shows a set of typical images gathered using the bubble tracer method. In this case some bubbles appear to be trapped in the interior of the circulating vortex and move vertically with the motion of the toroid. The vortex position is highlighted in the images shown by the arrows. Figure 3 also shows an enlargement (e) of a single image annotated to show the direction of motion of the vortex and individual bubbles which are seen to orbit the rings of fluid motion. This visualisation technique can be used to calculate the velocity of the vortex and the orbital motion of bubbles trapped in the circulating fluid. In this case the average velocity (over a $1 \mathrm{~mm}$ motion of the vortex) was found to be $20.2 \pm 4.1 \mathrm{~cm} \mathrm{~s}^{-1}$ (in reasonable agreement with the vortex shown in figure 2) while bubble velocities in this region were found to be of the order of $24.7 \pm 6.3 \mathrm{~cm} \mathrm{~s}^{-1}$. In addition to the advantage of imaging the velocity of bubbles trapped in the orbital motion of the vortex, the bubble tracer method can also be used to observe the repetitive behaviour of the vortex generation. In this case vortices were observed to be generated on each outward pulsation of the jet as expected. In these experiments the bubble tracer method has clear advantages over the single shot dye method. 
Returning to the dye method, as discussed earlier, this has been shown to be suited to the visualisation of the first fluid pulse but it is also useful in imaging the effects of the electrode substrate on the vortex motion in the system. This is an important factor as this pulsating jet approach has been suggested for use in HMV analysis. Figure 4 shows the initial generation of a vortex (note again only the first vortex is clear with this method), its subsequent motion through the liquid (see frames A4 to D4 for example) and finally, its impact on an electrode support. In this case a Pt electrode $(500 \mu \mathrm{m}$ diameter) embedded in a $0.45 \mathrm{~cm}$ diameter glass sheath was employed. However, several key differences can be gathered from these images. First, as the vortex approaches the solid/liquid interface of the electrode its diameter increases. This is as expected and has been documented in the literature[27]. Second, on impact with the solid/liquid support of the electrode, a set of vortices are generated. These secondary vortices (see ref[27] for example) can rotate in differing directions compared to the primary vortex[30]. The diameters of these vortices is clearly significantly larger than the primary vortex. For example the diameter $\left(d_{v}\right)$ of the primary vortex is $0.22 \mathrm{~cm}$ in frame A5 prior to the impingement on the surface of the electrode while the vortices shown in frame A6 have grown to $\sim 0.26 \mathrm{~cm}$ in diameter. The vortices shown in D8 have a diameter of the order of $0.53 \mathrm{~cm}$ significantly larger than the primary vortex that generated them. This is in agreement with other literature investigating the behaviour of vortices with a solid/liquid interface[27].

Finally it is of interest to link these visual observations with the electrochemical experiments reported previously[2]. Figure 5 shows a comparison of the electrode (see figure $5(a)$ ), the vortex generated by the apparatus (see image in figure 5 (b)) and the HMV data taken in the XZ plane of the apparatus (see figure 5 (c)). Figure 5 
(c) shows tracking of the mass transfer enhancement produced by the vortex as a function of the $X Z$ variable (the contour plot). Note this data has been amended from previous work[2] detailing mapping experiments using this apparatus and procedure. However, included in figure 5 (c) is tracking data showing the position of the centre of the lobes of the vortex $(\bullet)$.gathered from high-speed imaging experiments. The data shown in figure 5(b) and 5(c) shows a clear jet edge effect (denoted as a 'ring' in the $X Y$ plane previously[2] and labelled 'R' in figure 5(b)) associated with the jet perimeter and a further feature (labelled ' $\mathrm{H}$ ') when the jet edge crosses the edge of the glass electrode support (this was denoted as a 'halo' effect[2]). Clearly the region of mass transfer enhancement seen in the electrochemical data appears to coincide with the position of the vortex and its interaction with the electrode and the electrode substrate. In particular the 'ring' effect is associated with the position of the vortex itself which in turn is positioned at the edge of the jet mouth. This is detected when the vortex ring interacts with the electrode at 'F1' (see figure 5(a)). Lastly, the 'halo' effect noted previously[2] appears to coincide with the situation when the edge of the electrode support (see 'F2' on figure 5(a)) approaches the edge of the vortex ring itself[31] (see figure 5 (b), $Y$ displacement $\sim-2.3 \mathrm{~mm}$ ). Presumably under these circumstances either the vortex is no longer restricted by the presence of the solid/liquid interface of the electrode and its suppport (as previously noted when a vortex impacts on a solid/liquid interface) or it interacts with the edge of the electrode support causing an enhanced rate of mass transfer at the electrode itself. Clearly under these conditions an increase in the local mass transfer experienced by the electrode is detected. These findings, and those described previously[1-3], pertain to the pulsating flow induced in this experimental set up and 
the periodic vortex that is generated. However, they are also of interest to other experimental arrangements. For example Unwin et al. also noted an unexpected ring effect $[32,33]$ when using a microjet [34] electrode. In this case the fluid flow was maintained (as opposed to pulsed, as described here). In later work the flow patterns from an impinging jet were imaged[26] and simulated[35, 36] with vortex rings (under steady flow conditions) discussed[36]. 


\section{Conclusions}

The results shown here clearly demonstrated that the pulsating jet apparatus gives rise to the generation of a repetitive vortex ring which travels through the liquid and impinges on the surface of the electrode. This vortex has a Re value of the order of 1200 in free liquid and is observed to slow on approach to the electrode substrate. On impingement with the electrode/electrode support, further enlargement of the vortex was observed with the consequent generation of secondary/tertiary vortices. Finally the position, size of the vortex generated and the electrode support appears responsible for the ring and halo effect described previously[2].

\section{Acknowledgements}

We thank RSC/EPSRC Analytical studentship for funding JK under grant EP/C011430/1 and the EPSRC (EP/D05849X/1) for funding for the high-speed camera system. 


\section{Figure legends}

Figure $1 \mathrm{~A}$ schematic representation of a section of the pulsating jet apparatus and the position of the $50 \mu \mathrm{m}$ diameter Pt microwire electrode for electrochemical bubble generation. If dye experiments where studied, the inner volume of the inverted funnel was filled with the dye stained water while the outer volume was pure water whilst for the bubble tracking experiments the inner and outer aqueous solution contained $\left.0.1 \mathrm{~mol} \mathrm{dm}^{-3} \operatorname{Sr}\left(\mathrm{NO}_{3}\right)_{2}\right)$. The air/water interface is also labelled and the coordinate system used to describe the motion of the electrode in mapping experiments is shown for reference.

Figure 2 (a) Images showing the formation of the vortex during the initiation of jet oscillation captured using the dye technique. In this case the capture rate was 500 frames $\mathrm{s}^{-1}$ (inter frame time $2 \mathrm{~ms}$ ) and the first frame is at $4 \mathrm{~ms}$ after initiation of the transducer. The frames read left to right (A to D) and top to bottom (1-5). The times in parenthesis are shown in ms with respect to image A1. The horizontal line at the top of each image corresponds to the air/liquid interface. The jet exit is labelled ' $\mathrm{J}$ '. (b) Enlargement of a single image showing the development of the vortex and the 'neck' of dye leading back to the jet. The lower arrows indicate where fresh dye free liquid is being drawn back into the jet entrance. The inner diameter of the jet was 2 $\mathrm{mm}$ and can be used as a scale in each image.

Figure 3 Images (a) - (d) show individual high-speed images of a vortex recorded at 2000 frames $\mathrm{s}^{-1}$ with a shutter speed of $1 / 2500 \mathrm{~s}$. The actual times of the images selected in ms with respect to image (a) are shown in parenthesis. Oxygen bubbles were generated using a DC power supplier at a voltage of $5.1 \mathrm{~V}$ and current of 0.01 
A. The electrochemical cell used in the experiments consisted of $50 \mu \mathrm{m}$ diameter platinum wire electrode (see figure 1) and a platinum gauze counter electrode (not shown). The solution employed contained $0.1 \mathrm{~mol} \mathrm{dm}{ }^{-3} \operatorname{Sr}\left(\mathrm{NO}_{3}\right)_{2}$. A zero-to-peak piston displacement amplitude was $7.75 \times 10^{-3} \mathrm{~mm}$. Image (e) shows white arrows indicating the motion of the toroid and vortex within the image selected. The inner diameter of the jet was $2 \mathrm{~mm}$ and can be used as a scale in each image.

Figure 4 Sequence of images showing the vortex rising from a single pulse of liquid generated by the jet towards the electrode substrate. The electrode (labelled 'E' in image $\mathrm{A} 1$ ) was positioned $3 \mathrm{~mm}$ above the jet exit (labelled ' $\mathrm{J}$ ' in image $\mathrm{A} 1$ ). The capture rate was 500 frames $\mathrm{s}^{-1}$. The inter-frame time is $2 \mathrm{~ms}$ and the first frame is at $t=0 \mathrm{~ms}$. The frames read left to right $(A$ to $D)$ top to bottom (1-8). The times in parenthesis are shown in ms with respect to image $A 1$. The jet was oscillating at 16 Hz. The membrane cell was employed in the experiments and the zero-to-peak displacement amplitude of the membrane centre was $0.178 \pm 0.001 \mathrm{~mm}$.

Figure 5 (a) Image of a Pt electrode (500 $\mu \mathrm{m}$ diameter, labelled ' $\mathrm{Pt}$ ') in a glass support (5.5 mm diameter glass surrounding, labelled ' $G$ ') (b) Plot showing the current (o) recorded at the $\mathrm{Pt}$ electrode as a function of the displacement in $\mathrm{Y}$ direction (note $X=-0.2 \mathrm{~mm}$ in this case). The electrode was positioned $1 \mathrm{~mm}$ above the mouth of the jet (labelled ' $J$ '). The figure also includes an image of the vortex (for scale) generated under these conditions and highlighted using the dye method. The image is to scale with the electrochemical measurement. The annotation ' $R$ ' and ' $H$ ' denote the 'ring' and 'halo' effect described previously[2] (c) Plot showing the position on the ZX plane of the current enhancement recorded at a Pt electrode in a 
solution containing $0.1 \mathrm{~mol} \mathrm{dm}^{-3} \mathrm{Sr}\left(\mathrm{NO}_{3}\right)_{2}$ electrolyte containing $5 \mathrm{mmol} \mathrm{dm}{ }^{-3}$ $\left[\mathrm{Fe}(\mathrm{CN})_{6}\right]^{3-}$. Here the electrode was held under mass transfer limiting conditions and the legend shows the current in $\mu \mathrm{A}$. The $\left(^{\bullet}\right)$ symbol represents the trajectory of the vortex ring as a function of time (in the absence of the electrode) measured from high-speed imaging experiments. Note see figure 1 for $X Y Z$ orientation. 


\section{References}

[1] J. Kuleshova, P.R. Birkin, J.M. Elliott, Contribution of the Double Layer to Transient Faradaic Processes: Implications for Hydrodynamic Modulated Voltammetry of Nanostructures, Journal of Physical Chemistry C 114 (2010) 13442-50.

[2] J. Kuleshova, P.R. Birkin, J.M. Elliott, An HMV mapping study of a pulsating jet system, Journal of Electroanalytical Chemistry 617 (2008) 185-93.

[3] J. Kuleshova, P.R. Birkin, J.M. Elliott, Exploring the applications of a pulsating jet hydrodynamic modulated voltammetric (HMV) system - Electrochemistry of nanostructured Pt electrodes and trace analysis, Journal of Electroanalytical Chemistry 636 (2009) 68-73.

[4] D.E. Williams, K. Ellis, A. Colville, S.J. Dennison, G. Laguillo, J. Larsen, Hydrodynamic modulation using vibrating electrodes: Application to electroanalysis, Journal of Electroanalytical Chemistry 432 (1997) 159-69.

[5] D.E. Williams, J.V. Macpherson, Hydrodynamic Modulation Methods in Electrochemistry, in: R.G. Compton, G. Hancock (Eds), Comprehensive Chemical Kinetics - Applications of Kinetic Modelling, Elsevier, Amsterdame, 1999, pp. 369-438.

[6] W.J. Blaedel, G.W. Schieffer, Hydrodynamic Voltammetric Study of Ferricyanide-Ferrocyanide System with Convective Electrodes of Platinum, Gold, Glassy Carbon, Carbon-Film, and BoronCarbide, Journal of Electroanalytical Chemistry 80 (1977) 259-71.

[7] H.D. Dewald, B.A. Peterson, Ultrasonic Hydrodynamic Modulation Voltammetry, Analytical Chemistry 62 (1990) 779-82.

[8] J.V. Macpherson, Recent advances in hydrodynamic modulation voltammetry, Electroanalysis 12 (2000) 1001-11.

[9] J.V. Macpherson, P.R. Unwin, Hydrodynamic modulation voltammetry with a variable height radial flow microring electrode, Analytical Chemistry 71 (1999) 2939-44.

[10] B. Miller, S. Bruckenstein, Submicromolar analysis with rotating and hydrodynamically modulated disk electrodes, Analytical Chemistry 46 (1974) 2026-33.

[11] B. Miller, S. Bruckenstein, Theoretical and Experimental Study of Hydrodynamically Modulated Current-Potential Curves at Rotating-Disk Electrodes under Conditions of Mixed Electron and MassTransfer Control, Journal of the Electrochemical Society 121 (1974) 1558-62.

[12] B. Miller, S. Bruckenstein, Applications of Hydrodynamic Modulation at Rotating-Disk Electrodes to Semiconductor Electrochemistry, Annals of the New York Academy of Sciences 404 (1983) 473-4.

[13] S.A. Schuette, R.L. McCreery, Efficient Hydrodynamic Modulation with a Microcylinder Electrode, Analytical Chemistry 58 (1986) 1778-82.

[14] N. Simjee, P.R. Unwin, J.V. Macpherson, Hydrodynamic modulation voltammetry with a dual disk chopped flow-microjet electrode (CF-MJE), Electroanalysis 15 (2003) 1445-52.

[15] E.L. Cooper, L.A. Coury, Mass transport in sonovoltammetry with evidence of hydrodynamic modulation from ultrasound, Journal of the Electrochemical Society 145 (1998) 1994-9.

[16] C.R.S. Hagan, L.A. Coury, Comparison of Hydrodynamic Voltammetry Implemented by Sonication to a Rotating-Disk Electrode, Analytical Chemistry 66 (1994) 399-405.

[17] J.V. Macpherson, P.R. Unwin, Hydrodynamic modulation voltammetry with an oscillating microjet electrode, Analytical Chemistry 71 (1999) 4642-8.

[18] J. Kuleshova, An Invesitigation of Electrochemical Techniques in Acoustic Environments, University of Southampton, Southampton, 2008.

[19] G.S. Attard, P.N. Bartlett, N.R.B. Coleman, J.M. Elliott, J.R. Owen, J.H. Wang, Mesoporous platinum films from lyotropic liquid crystalline phases, Science 278 (1997) 838-40.

[20] J.M. Elliott, G.S. Attard, P.N. Bartlett, N.R.B. Coleman, D.A.S. Merckel, J.R. Owen, Nanostructured platinum (H-I-ePt) films: Effects of electrodeposition conditions on film properties, Chemistry of Materials 11 (1999) 3602-9.

[21] J.M. Elliott, P.R. Birkin, P.N. Bartlett, G.S. Attard, Platinum microelectrodes with unique high surface areas, Langmuir 15 (1999) 7411-5. 
[22] S. Ashforth-Frost, U.W. Rüdel, Thermal and Hydrodynamic Visualisation of a Water Jet Impinging on a Flat Surface using Microencapsulated Liquid Crystals, International Journal of Fluid Dynamics 7 (2002) 1-7.

[23] F. Sotiropoulos, D.R. Webster, T.C. Lackey, Experiments on Lagrangian transport in steady vortex-breakdown bubbles in a confined swirling flow, Journal of Fluid Mechanics 466 (2002) 215-48.

[24] D.R. Webster, E.K. Longmire, Vortex rings from cylinders with inclined exits, Physics of Fluids 10 (1998) 400-16.

[25] A.J. Smits, T.T. Lim, Flow Visualization: Techniques and examples, Imperial College Press, London, 2000.

[26] E. Bitziou, N.C. Rudd, M.A. Edwards, P.R. Unwin, Visualization and Modeling of the hydrodynamics of an impinging microjet, Analytical Chemistry 78 (2006) 1435-43.

[27] C.C. Chu, C.T. Wang, C.C. Chang, A vortex ring impinging on a solid plane surface - Vortex structure and surface force, Physics of Fluids 7 (1995) 1391-401.

[28] T. Seno, S. Kageyama, R. Ito, A Modeling of Vortex Rings in an Axisymmetric Pulsed Jet, Journal of Chemical Engineering of Japan 21 (1988) 1-5.

[29] A.J. Bard, L.R. Faulkner, Electrochemical Methods Fundamentals and Applications, 1st Ed. ed., John Wiley \& Sons, New York, 1980.

[30] J.K. Harvey, F.J. Perry, Flowfield Produced by Trailing Vortices in the Vicinity of the Ground, American Institute of Aeronautics and Astronautics 9 (1971) 1659-60.

[31] H.J. Lugt, Vortex flow in nature and technology, John Wiley and Sons, New York, 1983.

[32] J.V. Macpherson, M.A. Beeston, P.R. Unwin, Imaging Local Mass-Transfer Rates within an Impinging Jet and Studies of Fast Heterogeneous Electron-Transfer Kinetics Using the Microjet Electrode, Journal of the Chemical Society-Faraday Transactions 91 (1995) 899-904.

[33] J.V. Macpherson, M.A. Beeston, P.R. Unwin, N.P. Hughes, D. Littlewood, Scanning Electrochemical Microscopy as a Probe of Local Fluif Flow Through Porous Solids - Applications to the Measurement of Convective Rates Through a Single Dentinal Tubule, Journal of the Chemical Society-Faraday Transactions 91 (1995) 1407-10.

[34] J.V. Macpherson, S. Marcar, P.R. Unwin, Microjet Electrode - a Hydrodynamic Ultramicroelectrode with High Mass-Transfer Rates, Analytical Chemistry 66 (1994) 2175-9.

[35] J.L. Melville, B.A. Coles, R.G. Compton, N. Simjee, J.V. Macpherson, P.R. Unwin, Hydrodynamics and mass transport in wall tube and microjet electrodes. Simulation and experiment for micrometerscale electrodes, Journal of Physical Chemistry B 107 (2003) 379-86.

[36] J.L. Melville, N. Simjee, P.R. Unwin, B.A. Coles, R.G. Compton, Hydrodynamics and mass transport in wall tube and microjet electrodes: Effect of vortex formation and cell geometry on limiting currents, Journal of Physical Chemistry B 106 (2002) 10424-31. 


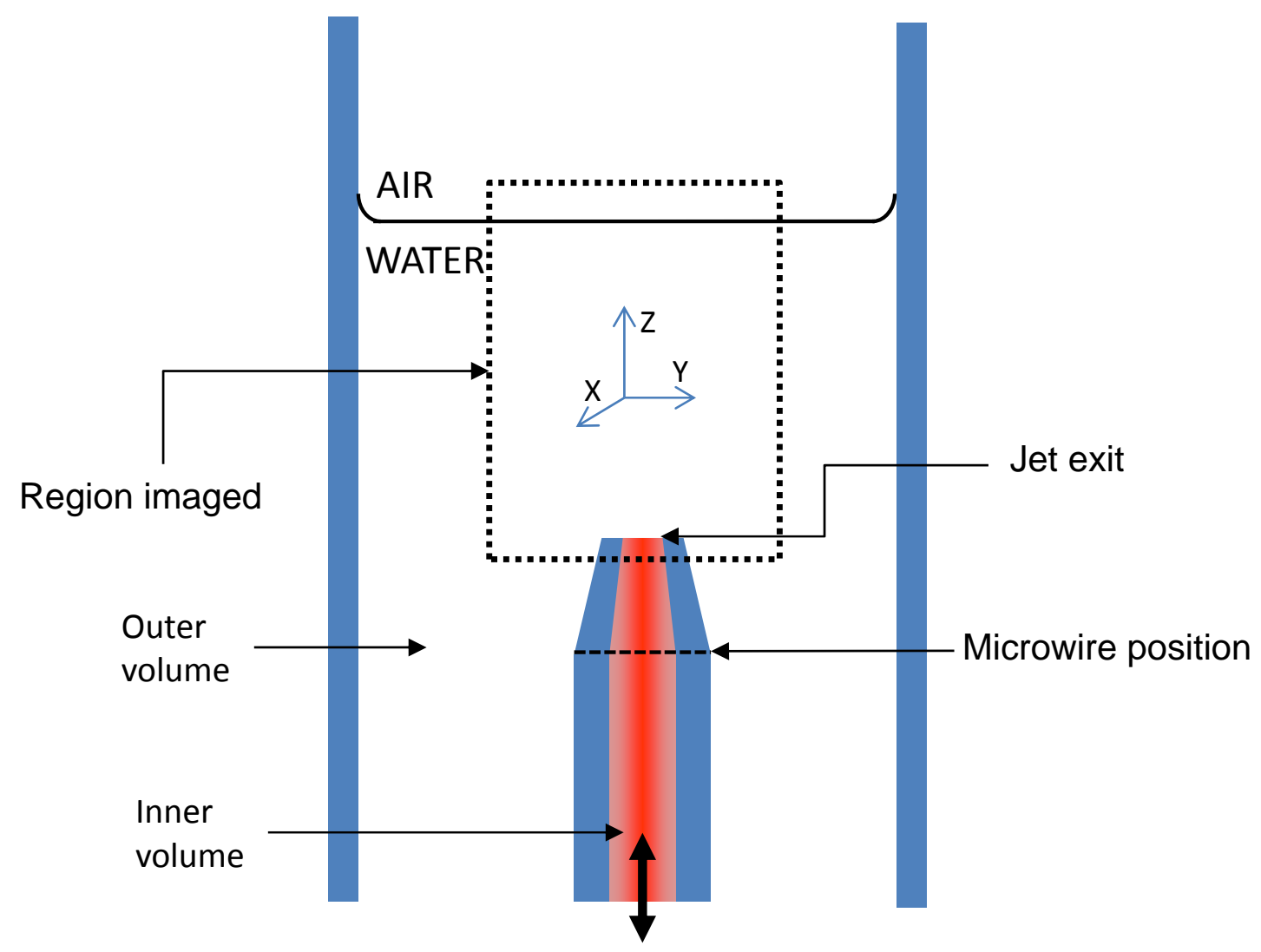

Fluid flow to and from

membrane/piston assembly

Figure 1 


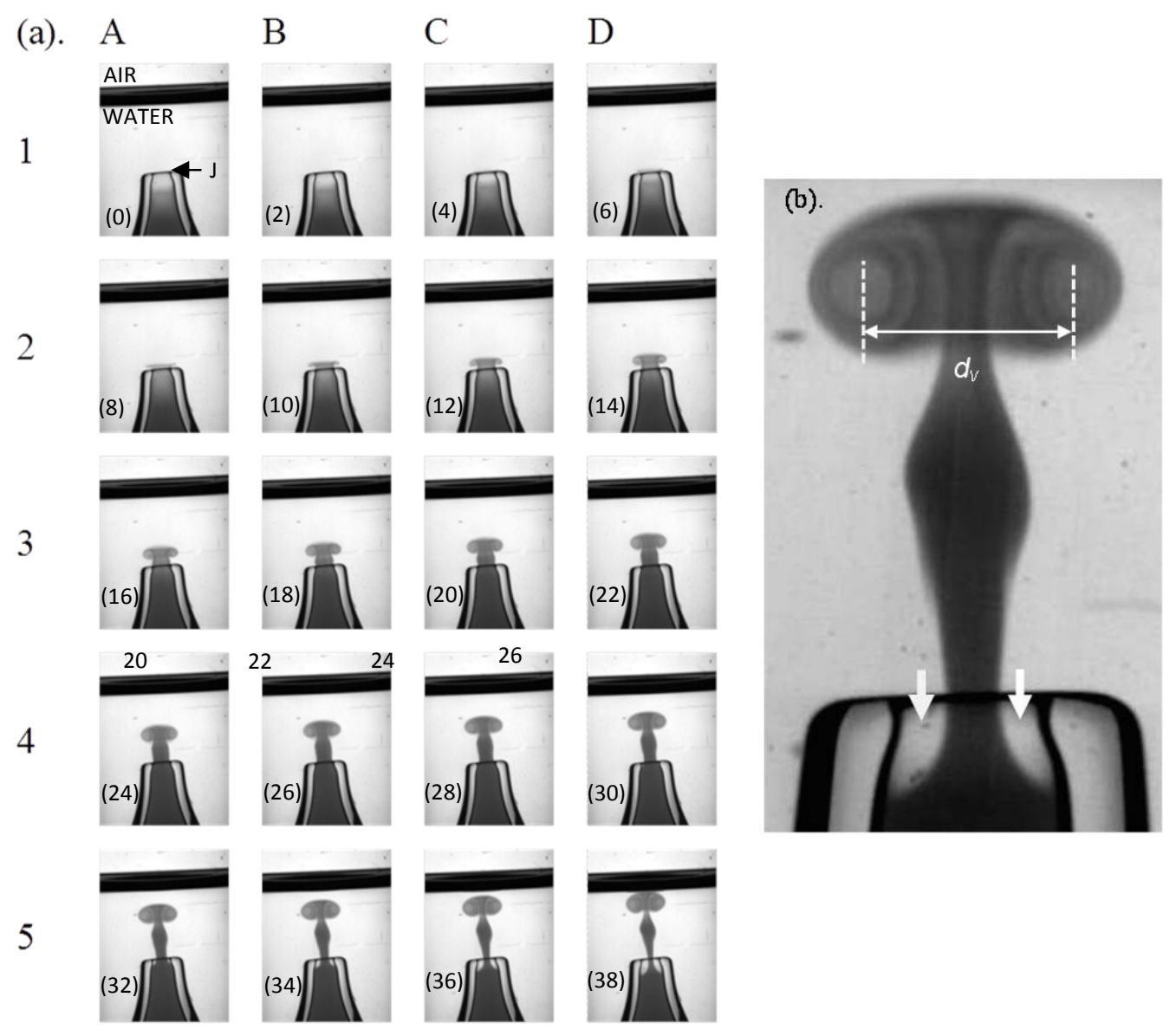

Figure 2 


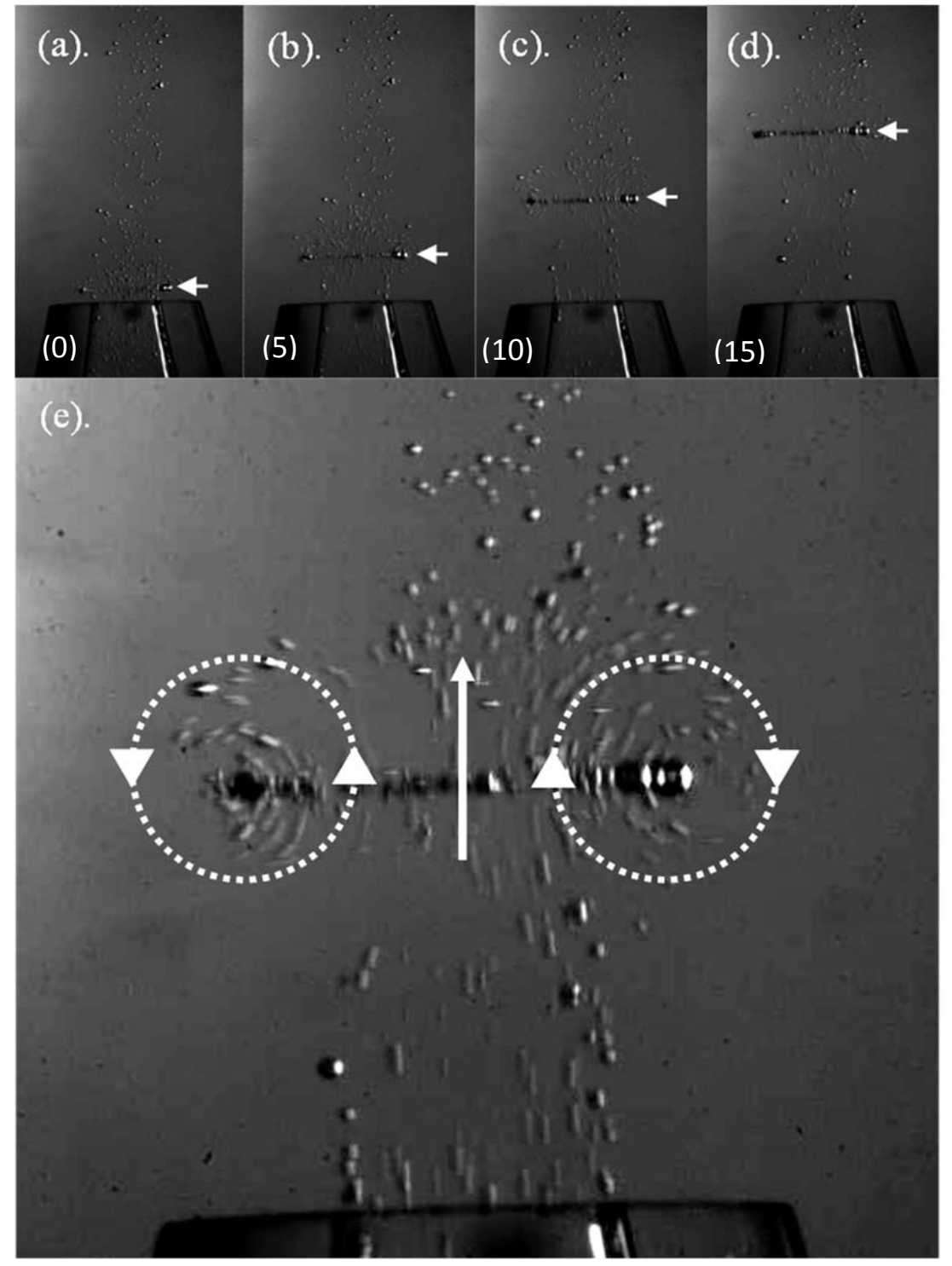

Figure 3 


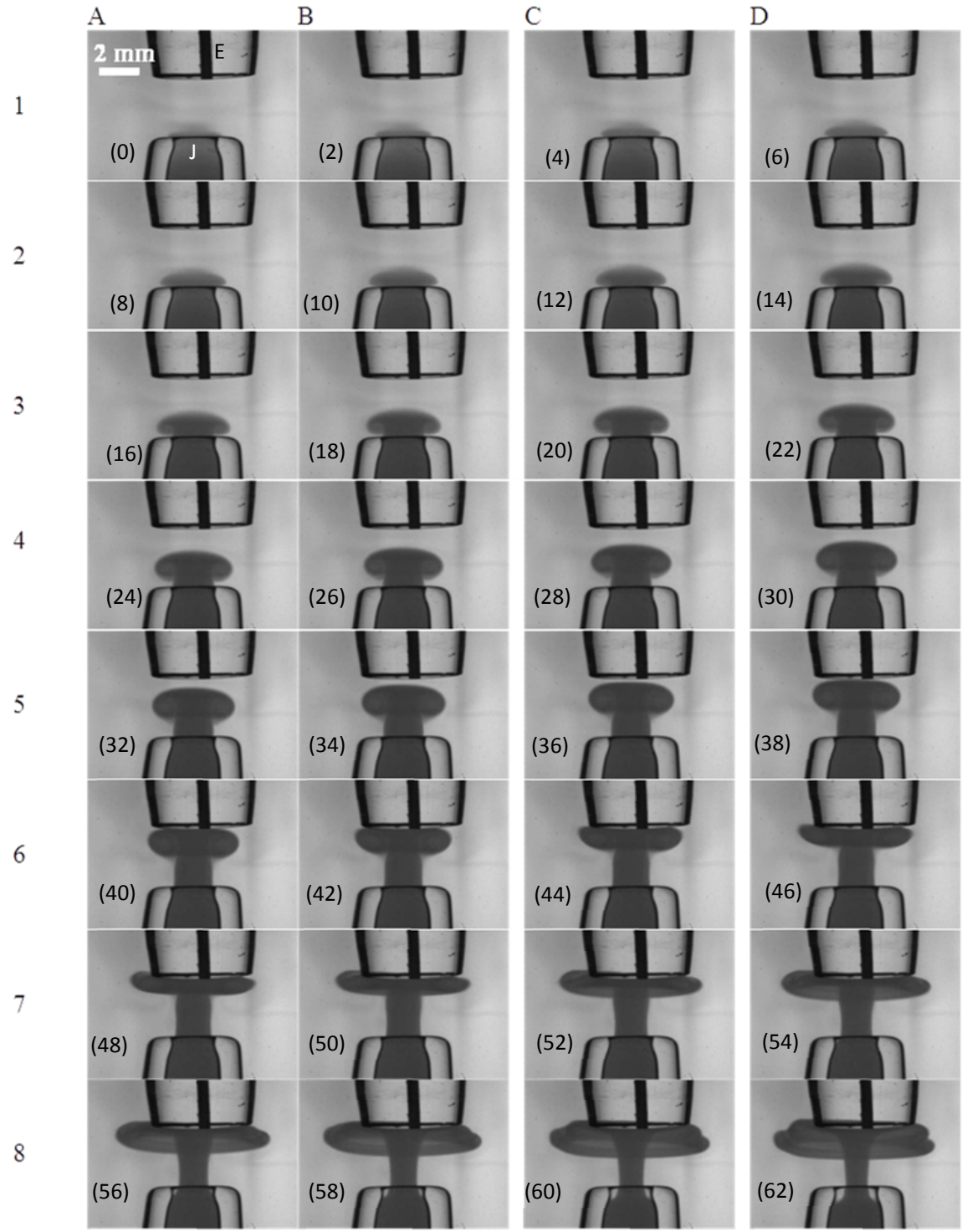

Figure 4 

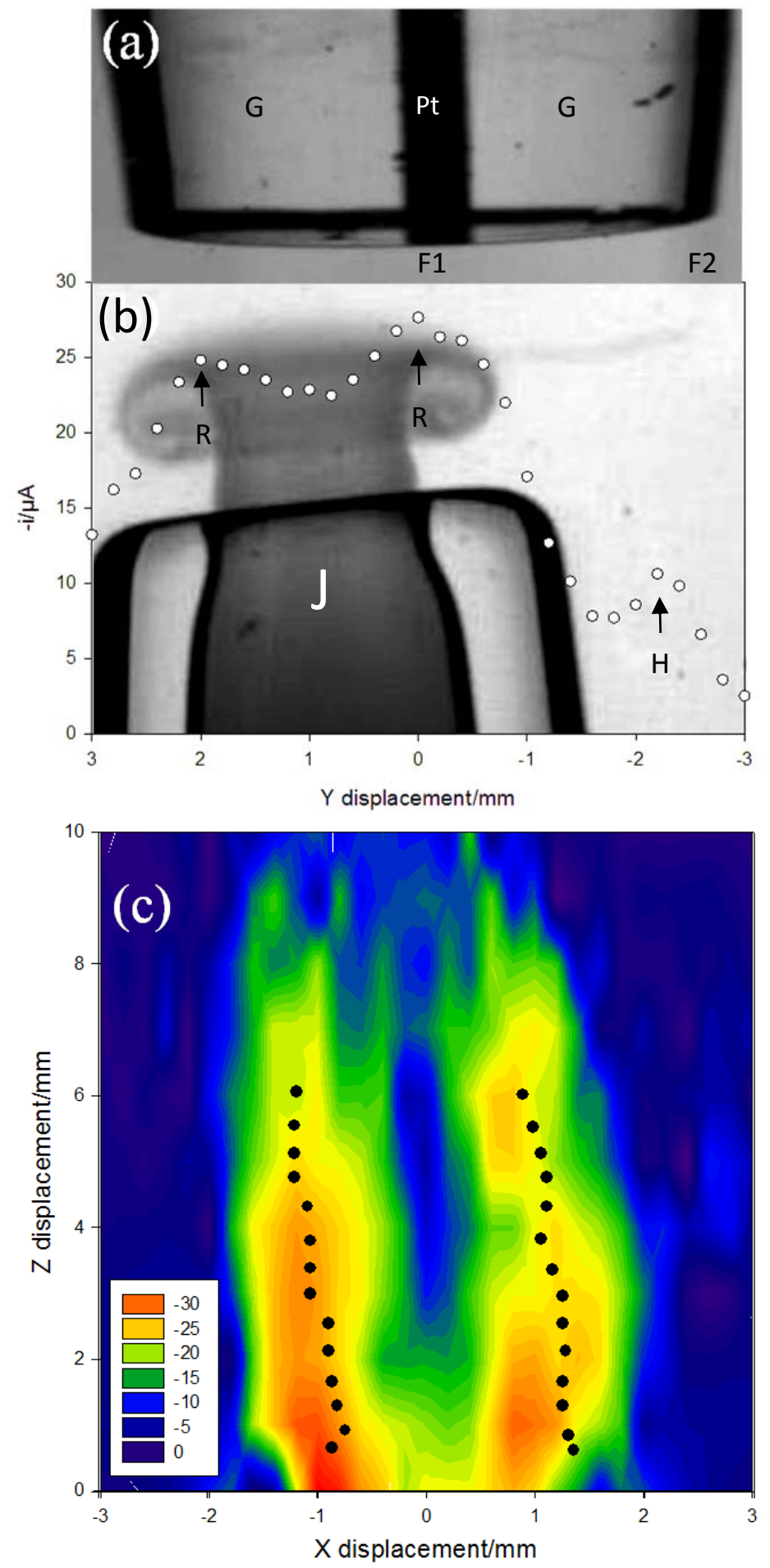

Figure 5 